\title{
Breast Metastasis of a Squamous Cell Carcinoma of the Uterine Cervix Mimicking Inflammatory Breast Cancer
}

\author{
Renaud Sabatier $^{a}$ Céline Roussin $^{e}$ Jean-Pierre Riviere $^{f}$ \\ Aurélie Jalaguier ${ }^{b}$ Jocelyne Jacquemier ${ }^{c}$ \\ François Bertucci ${ }^{a}$ d \\ Departments of ${ }^{\mathrm{a}}$ Medical Oncology, ${ }^{\mathrm{b}}$ Radiology and ${ }^{\mathrm{C}}$ Pathology, Institut \\ Paoli-Calmettes, and ${ }^{\mathrm{d}}$ University of Aix-Marseille, Marseille, ${ }^{\mathrm{e}}$ Department of \\ Medecine, Hôpitaux de Mayotte, Mamoudzou, and ' Department of Pathology, \\ Centre Hospitalier Félix Guyon, Saint-Denis, France
}

\section{Key Words}

Cervical cancer · Breast metastasis · Inflammation

\begin{abstract}
Breast metastases from distant carcinoma are infrequent, and cervix carcinoma is rarely the primary lesion. We describe the first case of a cervical squamous cell carcinoma with breast metastasis mimicking an inflammatory breast cancer in a 74-year-old woman. Seventeen months after the treatment of a primary tumor, the patient developed breast lesions looking like an inflammatory breast tumor. After a 1-year delay due to the patient's refusal, pathological examination and immunohistochemistry confirmed the diagnosis of breast metastasis from a poorly differentiated squamous cell carcinoma. The volume of the breast was huge, associated with axillary lymphadenopathies and multiple lung metastases. Despite platinum-based chemotherapy, the disease progressed and the patient died rapidly, 3 months after the first chemotherapy cycle and 15 months after the first mammary symptoms. We review the literature concerning breast metastases from gynecologic cancers and, particularly, from cervical squamous cell carcinoma. Differential diagnosis of such lesions may be problematic but is essential to avoid unnecessary mutilating surgery and to institute the appropriate systemic therapy. The prognosis is poor.
\end{abstract}




\section{Introduction}

Uterine cervical cancer is the most common primary cancer in women in emerging countries, with 454,000 cases and 200,000 deaths worldwide in 2010 [1]. Squamous cell carcinoma is the most frequent histological subtype. The pattern of metastatic diffusion initially involves pelvic lymph nodes, followed by para-aortic nodes and distant sites. The most frequent metastatic sites are the lungs, extra-pelvic nodes, liver, and bones [2]. However, due to increased survival thanks to multidisciplinary treatment, various other metastatic sites have been reported, including the breast. Mammary metastasis from cervical carcinoma is, nevertheless, extremely rare.

Here, we present the first case of breast metastasis from a cervical squamous cell carcinoma presenting clinically as an inflammatory breast cancer.

\section{Case Report}

The patient was a 74-year-old woman, African type, living in Mayotte (France, Indian Ocean). She had no specific medical personal or familial history, except for 9 pregnancies with 9 children. Her last delivery occurred in 1979. She was referred to a medical oncologist for the first time in March 2007 for pelvic pain starting 2 years prior to admission. Physical examination showed a large cervical tumor, with infiltration of the vaginal fornix and the parametrium. Her performance status was correct. A CT scan showed a huge cervical mass measuring $6 \times 7 \times 8 \mathrm{~cm}$, with involvement of the fornix, parametrium, mesorectum, and bladder. Cervical biopsies showed a poorly differentiated invasive squamous cell carcinoma. Two lung metastases ( 8 and $18 \mathrm{~mm}$ in the largest diameter) were observed. The diagnosis of FIGO stage IV cervical carcinoma was made. Fluorouracil- and platinumbased chemotherapy was thus started. After three cycles, the radiological evaluation showed a partial response with a decrease of the pelvic tumor and complete pulmonary response. We proposed to complete the treatment with external radiation therapy but the patient refused. After two more cycles of chemotherapy, the patient was lost to follow-up. She came back 3 months later in November 2007 because of abdominal pain and metrorrhagia. She accepted to start pelvic radiation therapy in February 2008. External conformational radiation therapy (45 Gy in 15 fractions) was delivered, allowing the complete disappearance of the clinical symptoms.

In August 2008, she presented with a small edema of the right breast with local irritation treated with antibiotics. One month later, her physician observed a right mastitis mimicking the clinical aspect of inflammatory breast cancer (swelling, edema, redness, and pain) associated with cutaneous permeation nodules and right axillary lymphadenopathies. A repeat CT scan showed lung metastases and cervical tumor recurrence, supraclavicular lymph nodes, and an enhancement of the fibroglandular density of the right breast without nodular lesion. At this time, the patient refused the diagnostic biopsy.

One year later, in August 2009, she accepted the biopsy because of the progressive breast involvement. The volume of her right breast was huge, 6-8-fold larger than that of the left breast. Multiple cutaneous permeation nodules were present ( $\underline{\text { fig. 1 }}$ ), associated with voluminous right axillary lymph nodes. A CT scan showed a diffuse skin thickening associated with permeation nodules, a large enhancement of the right breast, axillary lymph nodes, and lung metastases ( $\underline{\text { fig. } 2 \text { ). }}$.

Percutaneous biopsy of a permeation nodule on the right breast showed a subcutaneous infiltrate by a poorly differentiated squamous cell carcinoma consistent with a cervical origin, which matched the histology of the patient's primary tumor (ig. 3). Immunohistochemistry (IHC) showed CK7 negativity and strong P16 positivity, confirming the diagnosis of squamous cell carcinoma and eliminating the possibility of a metaplastic breast carcinoma. Platinum-based chemotherapy (weekly paclitaxel and carboplatin) was started. After three cycles, we observed a disease progression and proposed a new treatment with platinum and topotecan, which the patient refused. Best supportive care was set up. Breast, lymph nodes, and lung metastases kept growing, and the patient died a few weeks later, 3 months after the start of treatment for metastatic disease and 15 months after the appearance of the 
first breast symptoms. Written informed consent was obtained from the patient for publication of this case report and any accompanying images.

\section{Discussion}

The breast is an exceptional site of metastasis from cervical carcinoma. To our knowledge, the present case is the first report of metastatic squamous cell cervical cancer presenting clinically as an inflammatory breast cancer.

Metastatic involvement of the breast is very rare, representing $0.5-1.5 \%$ of all breast malignancies in clinical series and $6.6 \%$ in autopsy series $[3,4]$. The main primary cancers spreading to the breast are, in decreasing order of frequency, melanoma, lymphoma, lung cancer, soft tissue sarcoma, ovarian carcinoma, gastrointestinal cancers, and genitourinary tumors [5]. Ovarian cancer is the most frequent gynecologic cancer that metastasizes to the breast. The first cases of cervical carcinoma metastatic to the breast were described in autopsy series in 1947 [6], and the first case in a living patient in 1948 [7]. Since these cases, only few patients have been reported in the literature [8-14]. Distant metastases from cervical squamous cell carcinoma generally occur at the end of disease evolution. In most of the reported cases of breast metastasis, the patients had widespread disease with multiple other metastatic sites, notably lung metastases, as in our case.

The differential diagnosis between primary breast cancer and breast metastasis is crucial in order to avoid unnecessary breast surgery that can be as radical as a mastectomy and to deliver appropriate chemotherapy as soon as possible. However, it may be problematic. Clinically, the factor most suggestive of mammary metastasis is a history of cervical cancer. Indeed, clinical signs are non-specific. Most of the reported cases presented one or more palpable, mobile, painless, well-circumscribed breast nodule located in the subcutaneous tissues such as primary breast carcinoma. Skin modifications are generally absent. Clinical inflammatory presentation due to the involvement of superficial dermal lymphatic vessels and including tenderness, erythema, pain, and induration has never been reported so far for primary squamous cell cervical cancer, but has been reported for other primary cancers metastatic to the breast, e.g. ovarian cancer $[15,16]$, prostate cancer [17], cervical adenocarcinoma [9, 18], adenosquamous carcinoma [10], and lung adenocarcinoma [18]. Diffuse skin invasion and cutaneous permeation nodules have been reported with breast metastasis from melanoma [19]. Our case combined the inflammatory aspect and the presence of multiple permeation nodules. Axillary lymph node involvement is frequent, as observed in our patient.

The classical presentation of breast metastasis on mammography is a round lesion with smooth margins, without spiculation. Generally, and in contrast to primary breast carcinoma, metastatic breast lesions have the same radiological and clinical sizes because of a minimal desmoplastic reaction around them. Calcifications are uncommon except in metastasis of ovarian cancers [20,21]. Benign lesions such as fibroadenomas, galactoceles, and cysts are also possible differential diagnoses. Primary breast carcinomas to be considered with such mammographic findings are intra-cystic papillary, mucinous, and medullary carcinomas. Breast metastases are often multiple or bilateral lesions, localized at the upper outer quadrant, close to the skin. They grow 
rapidly and, when they are close to the skin, may induce skin edema or erythema, mimicking inflammatory breast cancer [22]. The common ultrasound feature of breast metastasis is an oval, hypoechoic or hyperechoic mass with a circumscribed or indistinct margin. A posterior acoustic enhancement is seen in $75 \%$ of cases due to fairly abundant cellularity [11]. Because breast metastases have often benign imaging features, any newly developed breast mass detected in a patient with a past history of extra-mammary malignancy requires radiological information and histological confirmation. MRI has been used to evaluate the extension of breast metastases and may be useful in young patients with dense breast parenchyma. Classically, CT scans show increased multiple and bilateral circumscribed masses associated with axillary lymph nodes. In case of breast metastasis mimicking breast inflammatory disease, as in our case, CT demonstrates a diffuse skin thickening with a large enhancement of the breast [23].

Histological diagnosis is thus mandatory. It can be performed by fine needle aspiration cytology [13] as well as biopsy. Morphological assessment is crucial, strongly helped by the clinical history and often by the IHC profile [18]. In our case, a typical aspect of invasive carcinoma rising from the squamous cells of the ectocervix was observed, and was associated with keratinization. IHC is poorly informative regarding cervical carcinoma as no specific marker exists. Breast metastases do not express estrogen and progesterone receptors or HER2 protein. Expression of CK7 and absence of expression of CK20 are present in half of cervical squamous cell carcinomas as well as primary breast cancers, notably metaplastic carcinomas, the main differential histological diagnosis [24]. In our patient, the negativity of CK7 and the strong positivity of P16 helped the diagnosis [25].

The prognosis of breast metastasis is poor as it implies widespread tumor dissemination. Most patients die within the year following the diagnosis [5, 8, 14]. Treatment is palliative, based on systemic therapy tailored to the primary cancer, sometimes completed by comfort loco-regional treatment of the breast lesion.

\section{Conclusion}

We report the first case of breast metastasis from a cervical squamous cell carcinoma presenting as an inflammatory breast cancer. Diagnosis was based on converging clinicoradiological and pathological arguments, notably the history of cervical cancer. Differential diagnosis is crucial to deliver the most appropriate treatment and should be systematically considered in a patient with a personal cancer history.

\section{Acknowledgements}

Our work is supported by the Institut Paoli-Calmettes and Aix-Marseille University.

\section{Disclosure Statement}

The authors declare that they have no conflict of interest. 


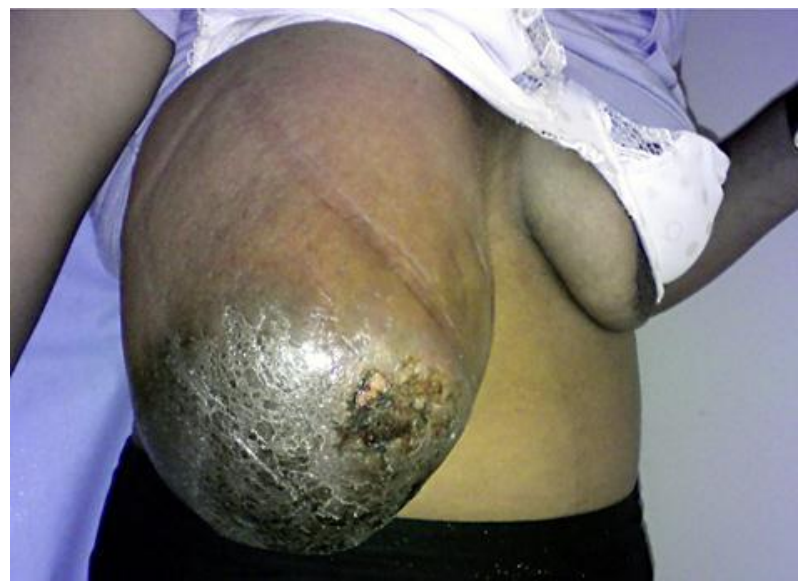

Fig. 1. Clinical aspect mimicking inflammatory breast cancer. A voluminous edema with skin infiltrate and cutaneous ulcerations is observed.
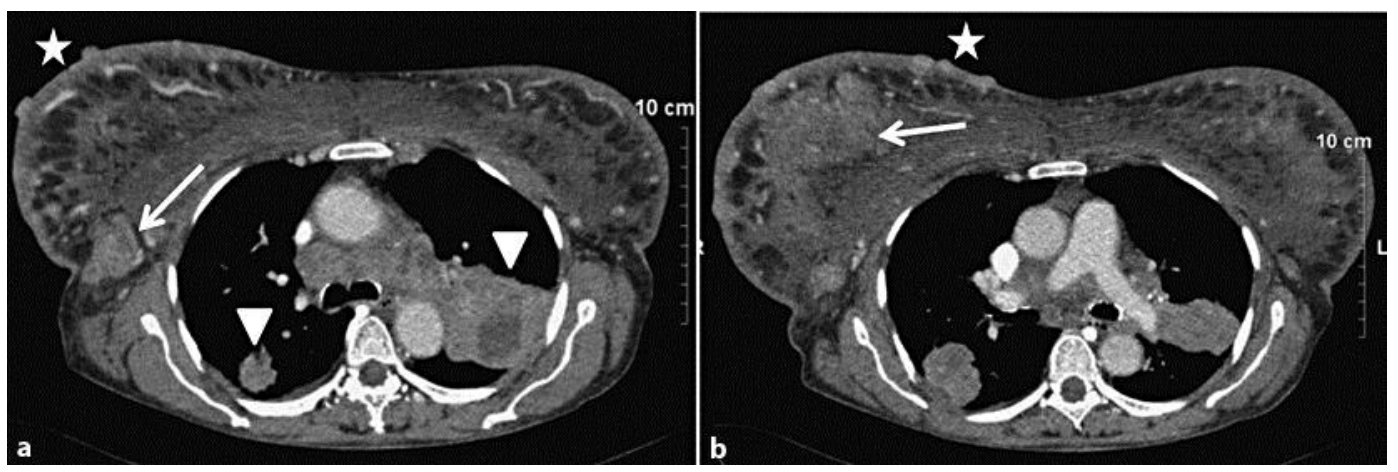

Fig. 2. CT scan features. a Axial contrast-enhanced section showing diffuse skin thickening (star) with bilateral lung metastases (arrowhead) and axillary lymph node (arrow). b The right breast is enlarged with a diffuse skin thickening with permeation nodules (star) and a large enhancement of the fibroglandular density without a well-defined nodular lesion (arrow). 

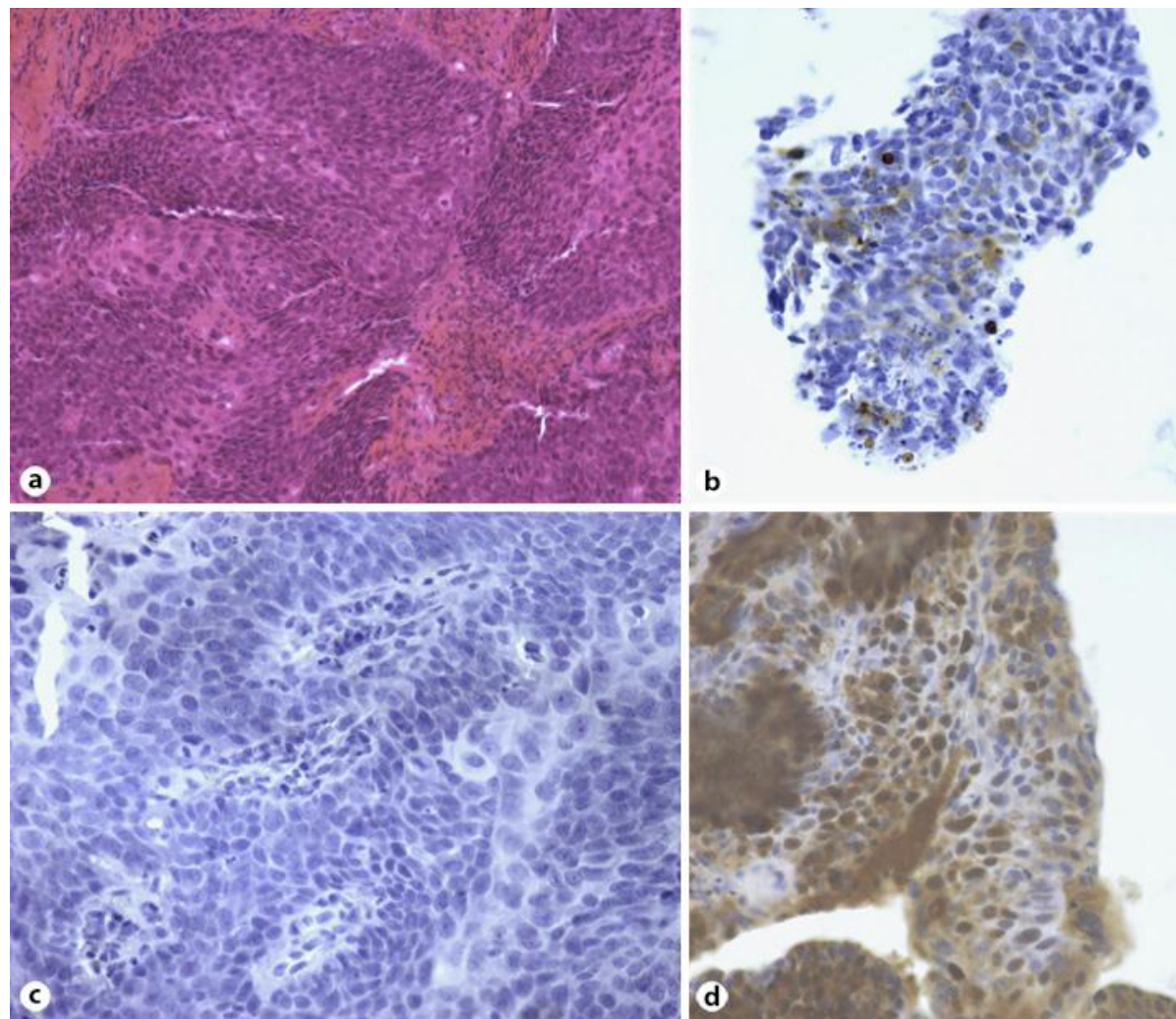

\section{b}

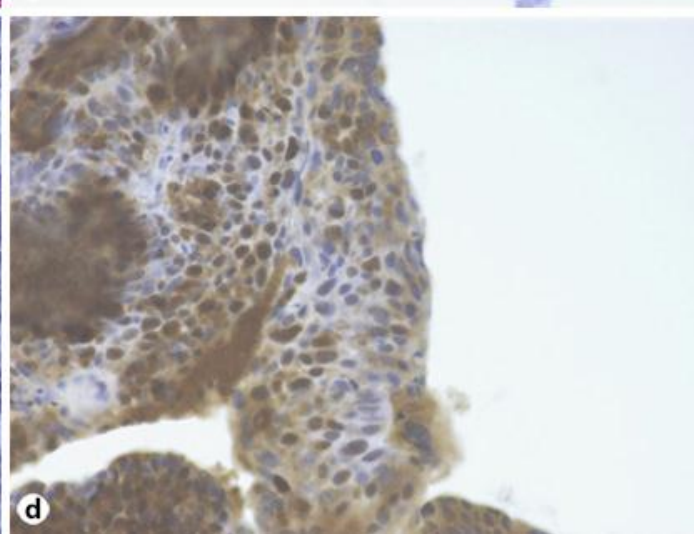

Fig. 3. Microscopic features. a Homogeneous aspect of squamous cell carcinoma with area of keratinization (HE staining). IHC showing heterogeneous expression of CK5/6 (b), absence of expression of CK7 (c), and strong positivity of P16 (d) (original magnification $\times 200$ ).

\section{References}

1 Forouzanfar MH, Foreman KJ, Delossantos AM, Lozano R, Lopez AD, Murray CJ, Naghavi M: Breast and cervical cancer in 187 countries between 1980 and 2010: a systematic analysis. Lancet 2011;378:14611484.

-2 Waggoner SE: Cervical cancer. Lancet 2003;361:2217-2225.

-3 Amichetti M, Perani B, Boi S: Metastases to the breast from extramammary malignancies. Oncology 1990;47:257-260.

4 Hajdu SI, Urban JA: Cancers metastatic to the breast. Cancer 1972;29:1691-1696.

5 Akçai MN: Metastatic disease in the breast. Breast 2002;11:526-528.

6 DeAlvarez RR: The causes of death in cancer of the cervix uteri. Am J Obstet Gynecol 1947;54:91-96.

7 Speert H, Greeley AV: Cervical cancer with metastasis to breast. Am J Obstet Gynecol 1948;55:894-896.

-8 Kumar L, Pokharel YH, Dawar R, Thulkar S: Cervical cancer metastatic to the breast: a case report and review of the literature. Clin Oncol (R Coll Radiol) 1999;11:414-416.

$\checkmark 9$ Ward R, Conner G, Delprado W, Dalley D: Metastatic adenocarcinoma of the cervix presenting as an inflammatory breast lesion. Gynecol Oncol 1989;35:399-405.

10 Kelley JL 3rd, Kanbour-Shakir A, Williams SL, Christopherson WA: Cervical cancer metastatic to the breast: a rare presentation of tumor dissemination. Gynecol Oncol 1991;43:291-294.

11 Younathan CM, Steinbach BG, DeBose CD: Metastatic cervical carcinoma to the breast. Gynecol Oncol 1992;45:211-213. 
12 Van Ooijen B, Slot A, Henzen-Logmans SC, Wiggers T: Cervical cancer metastasising to the breast: report of two cases. Eur J Surg 1993;159:125-126.

13 Gupta S, Gupta MK, Gupta R, Mishra RS: Breast metastasis of cervical carcinoma diagnosed by fine needle aspiration cytology. A case report. Acta Cytol 1998;42:959-962.

14 Moore DH, Wilson DK, Hurteau JA, Look KY, Stehman FB, Sutton GP: Gynecologic cancers metastatic to the breast. J Am Coll Surg 1998;187:178-181.

15 Kayikçioğlu F, Boran N, Ayhan A, Güler N: Inflammatory breast metastases of ovarian cancer: a case report. Gynecol Oncol 2001;83:613-616.

16 Gingell D, Samuel A, Haynik D, McBee W, Kelley J, Zorn K, Bhargava R: Metastatic ovarian serous carcinoma presenting as inflammatory breast cancer: a case report. Int J Gynecol Pathol 2010;29:243247.

17 Njiaju UO, Truica CL: Metastatic prostatic adenocarcinoma mimicking inflammatory breast carcinoma: a case report. Clin Breast Cancer 2010;10:E3-E5.

18 Fulciniti F, Losito S, Botti G, Di Mattia D, La Mura A, Pisano C, Pignata S: Metastases to the breast: role of fine needle cytology samples. Our experience with nine cases in 2 years. Ann Oncol 2008;19:682-687.

19 Chaignaud B, Hall TJ, Powers C, Subramony C, Scott-Conner CE: Diagnosis and natural history of extramammary tumors metastatic to the breast. J Am Coll Surg 1994;179:49-53.

20 Vizcaíno I, Torregrosa A, Higueras V, Morote V, Cremades A, Torres V, Olmos S, Molins C: Metastasis to the breast from extramammary malignancies: a report of four cases and a review of literature. Eur Radiol 2001;11:1659-1665.

21 Deshpande AH, Munshi MM, Lele VR, Bobhate SK: Aspiration cytology of extramammary tumors metastatic to the breast. Diag Cytopathol 1999;21:319-323.

-22 Feder JM, de Paredes ES, Hogge JP, Wilken JJ: Unusual breast lesions: radiologic-pathologic correlation. Radiographics 1999;19:S11-S26.

23 Yang WT, Le-Petross HT, Macapinlac H, Carkaci S, Gonzalez-Angulo AM, Dawood S, Resetkova E, Hortobagyi GN, Cristofanilli M: Inflammatory breast cancer: PET/CT, MRI, mammography, and sonography findings. Breast Cancer Res Treat 2008;109:417-426.

24 Chu P, Wu E, Weiss LM: Cytokeratin 7 and cytokeratin 20 expression in epithelial neoplasms: a survey of 435 cases. Mod Pathol 2000;13:962-972.

25 Thomas J, Primeaux T: Is p16 immunohistochemistry a more cost-effective method for identification of human papilloma virus-associated head and neck squamous cell carcinoma? Ann Diagn Pathol 2012;16:91-99. 